\title{
Asthma and epididymitis: the calm before the storm
}

Gerald H M George, John S Axford

\section{Case report}

A 17 year old Asian college student presented with a two month history of arthralgia, arthritis of his ankles and wrists, numbness of his feet and weight loss. He had a history, for the previous month, of epididymo-orchitis treated at another hospital.

He had been investigated for abdominal pain and weight loss by plain abdominal radiography, abdominal ultrasound scan and rectal biopsy. Results were all normal. The symptoms were associated with a poor appetite but no history of nausea, vomiting or diarrhoea. No definite diagnosis was reached.

He had a painful left ankle with associated numbness of the lateral aspect of his left foot. $\mathrm{He}$ felt generally weak and lethargic. He subsequently developed right ankle discomfort and swelling and bilateral wrist swelling and pain.

Past medical history included allergic rhinitis and asthma, since childhood, for which he was treated regularly with beclomethasone and salbutamol inhalers.

Clinical examination on presentation revealed a thin, unwell, feverish patient. $\mathrm{He}$ was $160 \mathrm{~cm}$ tall and weighed $43 \mathrm{~kg}$. His fever persisted during the early stages of his admission to hospital. Hand examination revealed splinter haemorrhages, macular pigmented lesions on the palmar surface of his hands, and subcutaneous nodules at both elbows. He had a tachycardia with a soft ejection systolic murmur and his blood pressure was normal. His chest was clear and abdomen soft and non-tender with no palpable organs or masses. Central nervous system examination was normal. Peripheral nervous system examination revealed an asymmetrical subjective light touch sensory diminution in the lower limbs below the knees and absent ankle jerk reflexes bilaterally. Plantar reflexes were both flexor. There was grade $3 / 5$ weakness of dorsiflexion at both ankles. Urine analysis was negative for blood and protein.

\section{Investigations}

Laboratory investigations showed: haemoglobin $15.0 \mathrm{~g} / \mathrm{dl}$, leucocyte count $21.5 \times 10^{9} / 1$, platelets $461 \times 10^{9} / 1$, erythocyte sedimentation rate (ESR) $146 \mathrm{~mm} 1 \mathrm{st} \mathrm{h}$, and C reactive protein (CRP) $37 \mathrm{mg} / \mathrm{l}$. Serum urea, creatinine, electrolytes, liver function tests, bone biochemistry, creatine kinase and angiotensin convert- ing enzyme were all normal. Rheumatoid factor was $490 \mathrm{iu} / 1$ (normal $<20 \mathrm{iu} / 1$ ), ANA weak positive, anti double stranded DNA and anti- ENA antibodies negative, and cANCA weak positive but negative for proteinase 3 and myeloperoxidase.

Further investigations were as follows: there was an eosinophilia of $14.8 \times 10^{9} / 1$. $\operatorname{IgE}$ was markedly raised with an initial level of $540 \mathrm{ku} / 1$ later peaking at $5118 \mathrm{ku} / 1$. Serial blood cultures were sterile. Trans-thoracic echocardiogram demonstrated a mass on the mitral valve chordae tendinae. Radiographs of hands, feet, chest and abdominal ultrasound scan were normal. Microscopy of initial left elbow nodule biopsy showed the presence of granulomatous tissue with necrosis. Subsequent skin biopsy from a subcutaneous nodule at the right shoulder was consistent with a leucocytoclastic vasculitis. EMG revealed an increased latency of the right and left lateral, and right medial popliteal knee to ankle motor nerve conduction with reduced amplitude. Right radial sensory, right ulnar and median motor nerve conduction were normal.

\section{Differential diagnosis}

1 Sub-acute bacterial endocarditis, in view of his fever, weight loss, murmur and echocardiogram findings. The mass on the mitral valve chordae tendinae was initially considered to be supportive of a diagnosis of infective endocarditis in keeping with the clinical context of presentation. However, after two subsequent echocardiograms, the lesion was deemed to be consistent with probable ruptured chordae tendinae. There was neither valvular regurgitation nor other evidence of infective endocarditis.

2 Rheumatoid arthritis with vasculitis, in view of his arthralgia, arthritis, nodules at both elbows, and positive rheumatoid factor. Initial skin biopsy was consistent with a rheumatoid nodule. The skin showed focal depigmentation with lymphocytes and histiocytic aggregates in the upper dermis, and some altered collagen. The leucocytoclastic biopsy specimen had features of patchy basal degeneration with occasional dyskaryotic cells in the epidermis. There was focal ulceration and the dermis showed perivascular and periadnexal active chronic inflammation with focal fibrinoid change in a vessel with prominent nuclear dust indicating a leucocytoclastic vasculitis. 
3 Other vasculitides such as Churg-Strauss syndrome (CSS), in view of asthma, allergic rhinitis, arthralgia, arthritis, and neurological findings.

\section{Evidence for CSS}

(1) The presence of asthma was the most discriminative criterion in segregating CSS from other forms of vasculitis in the ACR (American College of Rheumatology) criteria classification series. ${ }^{1}$ As in this case, asthma often precedes the other features of CSS by several years and up to as long as 30 years. $^{2}$ It commonly occurs in the fourth decade in life and its severity and frequency increases before the systemic manifestations of CSS. ${ }^{3}$ Remission of asthma often coincides with the onset of manifestations of systemic features of CSS but relapse may occur in the post-vasculitic phase. ${ }^{2}$

Allergic rhinitis, as in this case, sinusitis, and nasal polyps are common upper airways associations in CSS.

(2) Arthralgia and arthritis are common in CSS, may be flitting in nature, and tend to occur during the vasculitic phase of the illness as occurred in this case. Similarly myalgia is common but myositis is rare. ${ }^{134}$ Rheumatoid factor (RF) has been noted to be positive, usually in low titres but occasional high concentrations have been reported. ${ }^{2}{ }^{3}$ IgG and IgM serum RF have been observed to be transiently raised in the vasculitic phase of the illness. This coincides with a transient increase in the concentrations of interleukin 6 (IL6), suggesting that RF and IL6 may be important in the pathogenesis of the vasculitis of CSS. ${ }^{5}$

(3) A mononeuritis multiplex of the lower limbs was confirmed by EMG. Vascular neuropathy presents with either a mononeuritis multiplex or, symmetrical or asymmetrical sensori-motor neuropathy. In a review of vasculitis selectively affecting the peripheral nervous system, most had a mononeuritis multiplex or asymmetrical neuropathy and some had a symmetrical neuropathy. ${ }^{6}$ Mononeuritis multiplex is usually a necrotising vasculitis whereas a distal neuropathy is probably related to vasa nervorum micro-circulation occlusion.

Shintani reported a case of third nerve palsy and mononeuritis multiplex associated with CSS. ${ }^{7}$ Cranial nerve palsies are unusual in CSS and the most frequent cranial nerve lesion observed is an ischaemic optic neuritis. In this case there were no cranial nerve palsies but there was a mononeuritis multiplex confirmed by nerve conduction studies.

The traditional format ACR criteria for CSS lists neuropathy as being $75 \%$ sensitive and $79.8 \%$ specific for CSS against other defined vasculitis syndromes. The neuropathy may be a mononeuropathy, mononeuritis multiplex or polyneuropathy. Neuropathy lies third in the order of sensitivity in the diagnosis of CSS. ${ }^{1}$

(4) Examination of the differential leucocyte count revealed marked eosinophilia of $14.8 \times$ $10^{9} / 1$. Eosinophilia is essential to the diagnosis of CSS. However there are other situations where eosinophilia may be related to arthralgia, vasculitis and autoimmune rheumatic diseases. In a review of 265 British cases of Wegener's granulomatosis, eosinophilia was a frequent laboratory feature though it was not a prerequisite for diagnosis. ${ }^{8}$ Eosinophilia myalgia syndrome, which shares features with eosinophilic fasciitis and other variants of systemic sclerosis, is characterised by peripheral blood eosinophilia. ${ }^{9}$ Blood eosinophilia may be present in localised scleroderma and coincides with active disease. ${ }^{10}$ Eosinophilia has been reported in rheumatoid arthritis, both related and unrelated to drug treatment. ${ }^{112}$ In some cases eosinophilia has been suggested as a marker of extra-articular manifestations of rheumatoid arthritis. ${ }^{13}$ On a rarer note, Espino reported a case of eosinophilic myositis associated with vasculitis and symmetrical polyneuropathy, presenting as myalgia, muscle weakness and eosinophilia. ${ }^{14}$ The pulmonary eosinophilias, reviewed elsewhere, ${ }^{15-17}$ are a diverse group of disorders characterised by pulmonary infiltrates, rich in eosinophils and usually associated with a peripheral blood eosinophilia.

In the ACR classification criteria for CSS, the presence of eosinophilia was the second most useful criterion in segregating CSS from other forms of vasculitis. ${ }^{1}$ This was further specific if the eosinophilia was $>10 \%$ on a white blood cell differential smear as occurred in most patients. An eosinophilia of $>10 \%$ was $95 \%$ sensitive and $96.6 \%$ specific for CSS compared with other defined vasculitis syndromes. The current reported case had an eosinophilia of $>10 \%$. This value varied with disease activity, normalising with clinical remission of disease activity.

(5) The $\operatorname{IgE}$ was markedly raised. In the series of patients recruited by the ACR to create criteria for the classification of CSS, IgE concentrations were raised in all patients in whom it was measured. Concentrations ranged from 184-2200 units $/ \mathrm{ml}^{1}{ }^{1}$ Lanham et al observed raised $\operatorname{IgE}$ in $75 \%$ of patients, both in their series and in the accompanying literature search review. ${ }^{3}$ This was particularly so in the vasculitic phase of CSS with normal concentrations in remission. Raised IgE concentrations were observed in this case report, and $\operatorname{IgE}$ is implicated in the pathogenesis of asthma and allergic rhinitis as well as vasculitis. ${ }^{18}$

(6) The skin and histology findings were supportive of CSS. Seventy per cent of patients with CSS have skin involvement. ${ }^{319}$ Any combination of papules, macules, nodules and papulovesicles may occur. Three categories of lesions are recognised: erythematous maculopapules resembling erythema multiforme (40\%); haemorrhagic lesions ranging from petechiae to often palpable extensive ecchymoses (45\%); cutaneous and subcutaneous nodules $(20 \%)$, often tender, persistent and resolve with scarring. Nodules histologically show the characteristic granuloma of CSS. An eosinophilic infiltrate progresses into granuloma formation, beginning with necrosis within a densely packed cluster of eosinophils. The necrotic core enlarges with inclusion of collagenous stroma, and epithelioid and giant cells appear around the core to produce a palisading formation several cells thick. The type of 
granuloma seen in CSS may be seen in other disorders, including rheumatoid arthritis, polyarteritis nodosa, systemic lupus erythematosus, sub-acute bacterial endocarditis, lymphoproliferative disorders and chronic active hepatitis. ${ }^{20}$

Histological examination of the other skin lesions in CSS is usually of a small vessel leucocytoclastic vasculitis, with a predominance of neutrophils. The second biopsy in this case was consistent with a leucocytoclastic vasculitis.

\section{Other notable features of CSS relevant to this case \\ EPIDIDYMITIS}

It can only be assumed that the episode in this case may be related to the diagnosis of CSS. Epididymitis as a manifestation of ARD is rare. However, there have been several case reports and case series of this. Wright ${ }^{21}$ reported on two cases in which epididymitis heralded the onset of more severe visceral organ damage. In our case, the presentation of epididymitis was at a different hospital and so the precise aetiology is unknown. Shurbaji ${ }^{22}$ described nine cases of testicular vasculitis identified from surgical and necropsy files. In three cases this was the initial presentation of polyarteritis nodosa (PAN), one of these being as epididymitis. In two separate cases testicular vasculitis proceeded systemic PAN. There was one case of Goodpastures syndrome and three incidental findings without diseases associated with vasculitis. Kroegel $^{23}$ reported a case of granulomatous epididymitis occurring before the development of asthma and eosinophilia in CSS. The consensus of opinion would therefore be that epididymitis as a manifestation of CSS is rare.

CARDIAC INVOLVEMENT

Cardiac manifestations of CSS are best demonstrated by echocardiography. Mitral regurgitation is common. ${ }^{24}{ }^{25}$ In this case there was the presence of ruptured chordae tendinae, which may be a preceding factor in the progression to mitral regurgitation. Vasculitis of the coronary arteries may occur with all the expected consequences of this. ${ }^{25-27}$ Pericarditis and pericardial effusion may occur in $25 \%$ but these are not sensitive enough features to be included in the diagnostic criteria. ${ }^{1}$ There may be congestive cardiac failure or restrictive cardiomyopathy. ${ }^{28}$ Cardiac disease accounts for $48 \%$ of deaths attributable to CSS. ${ }^{3}$

Cardiac granulomas are commonly found at necropsy and cardiac failure featured in the series studied for the ACR criteria. ${ }^{1329}$ With the advent of corticosteroid and immunosuppressive treatment, improved diagnostic methods and awareness of cardiac involvement in CSS, the prognosis of CSS determined by cardiac disease should improve. ${ }^{30}$

GASTROINTESTINAL MANIFESTATIONS

Gastrointestinal involvement is frequent in CSS. Symptoms range from abdominal pain and diarrhoea, to haemorrhage and perfora- tion. The causes include eosinophilic infiltration of the bowel wall and mesenteric vasculitis, the former being rare. ${ }^{31}$

This case had been investigated for abdominal pain before presentation at our centre. There were no specific findings and the abdominal pain settled before presentation without further recurrence.

\section{RENAL DISEASE}

Renal disease in CSS is mild and rarely progresses to renal failure. ${ }^{23}$ This provides a distinguishing feature when compared with polyarteritis nodosa and Wegener's granulomatosis. In this case, renal function has remained normal throughout the duration of the disease. In the series studied by Lanham, $49 \%$ had mild or moderate renal disease but only $9 \%$ (one patient) had renal failure. ${ }^{4}$ Histologically the lesions vary. The original findings of Churg and Strauss were of focal glomerular lesions, a diffuse or focal interstitial nephritis, and necrotising vasculitis with granulomatous nodules. Lanham on the other hand found a focal segmental glomerulonephritis, with necrotising features, including crescents in some cases. The case of renal failure, in that series, reversed with corticosteroids and immunosuppressive treatment, demonstrating the less aggressive nature of renal disease as compared with patients with Wegener's granulomatosis. Necrotising glomerulonephritis in CSS are often associated with p-ANCA. ${ }^{32}$

\section{PRESENCE OF ANCA ANTIBODIES}

Anti-neutrophil cytoplasmic antibodies (ANCA) are neither specific nor sensitive in the diagnosis of CSS. ${ }^{33}$ However, CSS is strongly associated with ANCA, the frequency being approximately $50 \% .{ }^{34}$ In a study by Guillevin, $67 \%$ of patients with CSS had positive ANCA titres. Most were perinuclear ANCA (pANCA) positive. ${ }^{35}$ Others have found this association $^{36}{ }^{37}$ but the value of serial ANCA measurements for the monitoring of treatment efficacy has not yet been established. ${ }^{38}$ This case is unusual as a weak positive cytoplasmic ANCA (cANCA) staining pattern was obtained but with no detectable antibody to serine proteinase 3 (PR3). On repeated occasions in this case, ANCA staining was negative, suggesting the initial weak positive result may have been a non-specific finding associated with the inflammatory process especially in the light of the absence of antibodies to PR3.

\section{Initial management}

After investigations and the exclusion and treatment of possible infection, the diagnosis of Churg-Strauss syndrome was made. The patient was prescribed $30 \mathrm{mg}$ prednisolone daily and this was subsequently increased to $40 \mathrm{mg}$ daily. Azathioprine was added initially at $50 \mathrm{mg}$ daily and increased to a maintenance dose of $100 \mathrm{mg}$ daily (equivalent to $>2 \mathrm{mg} / \mathrm{kg} /$ day) to consolidate treatment of his symptoms of arthralgia and lethargy, and as a corticosteroid sparing agent. There was subsequent improvement in his overall condition and the patient was discharged from hospital. 

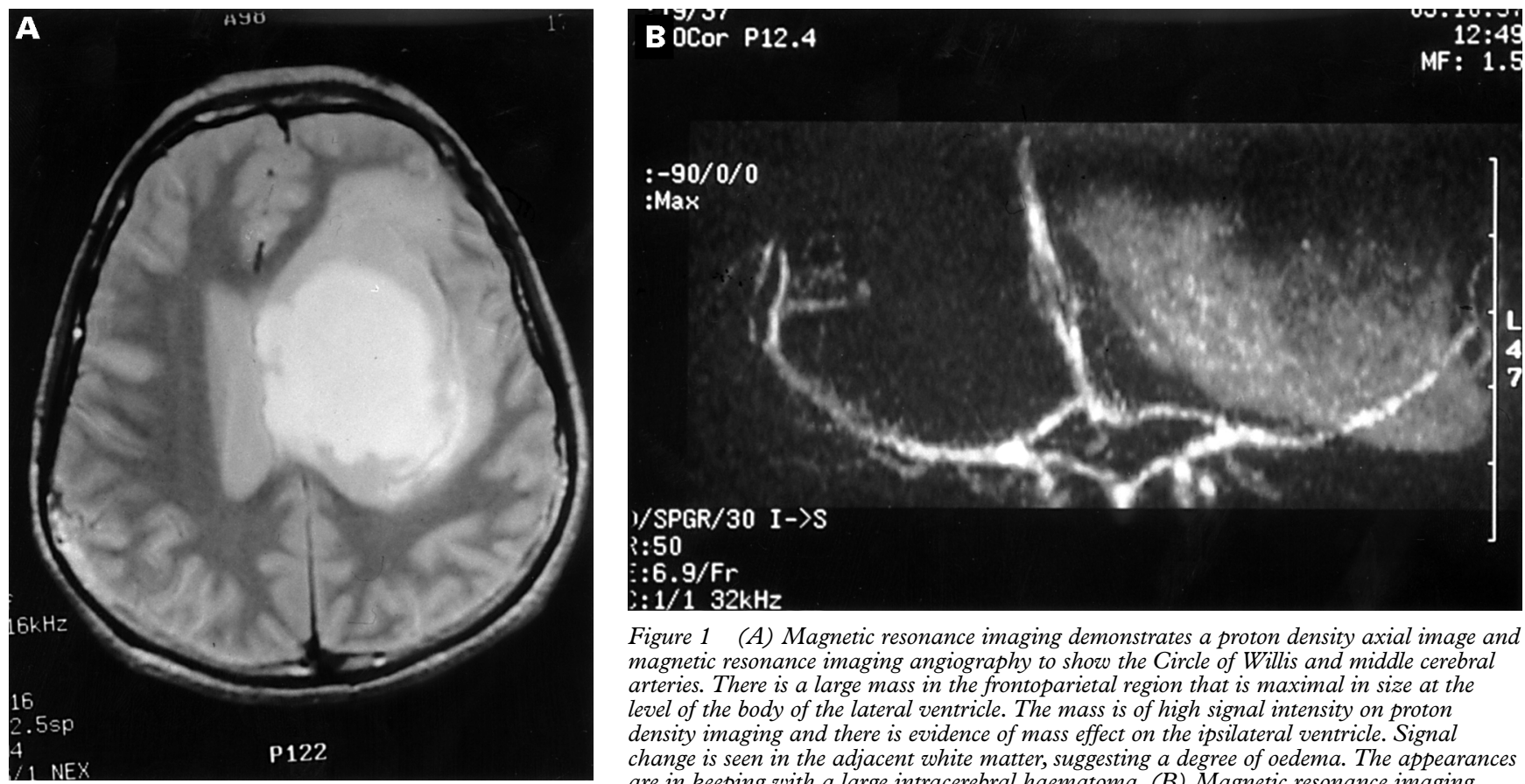

Figure 1 (A) Magnetic resonance imaging demonstrates a proton density axial image and magnetic resonance imaging angiography to show the Circle of Willis and middle cerebral arteries. There is a large mass in the frontoparietal region that is maximal in size at the level of the body of the lateral ventricle. The mass is of high signal intensity on proton density imaging and there is evidence of mass effect on the ipsilateral ventricle. Signal change is seen in the adjacent white matter, suggesting a degree of oedema. The appearances are in keeping with a large intracerebral haematoma. (B) Magnetic resonance imaging angiogram shows displacement of vessels secondary to the haematoma.

Subsequent presentation

Six months after presentation the patient developed sudden onset headache, nausea, vomiting and deteriorating level of consciousness. He had a Glasgow coma score of $9 / 15$. Eye opening scored $2 / 4$, verbal response $2 / 5$ and motor response 5/6. Motor response localised on the left side and flexed on the right side. Pupils were $4 \mathrm{~mm}$ and non-reactive to light. Blood pressure was 130/80. Computed tomography of the head scan showed a large left sylvian fissure intra-cerebral haemorrhage extending to the left frontal lobe, left lateral ventricle and 3rd ventricle. Laboratory investigations at this time showed: Haemoglobin 10.3 $\mathrm{g} / \mathrm{dl}$, leucocyte count $16.8 \times 10^{9} / 1$, eosinophils $1 \times 10^{9} / 1$, ESR $104 \mathrm{~mm} \mathrm{1st} \mathrm{h,} \mathrm{CRP} 105 \mathrm{mg} / 1$ Urea and electrolytes were normal. On this occasion IgE was not measured.

\section{Subsequent management}

The patient required intubation, ventilation and a frontal ventriculostomy. A cerebral angiogram at the time showed no evidence of arteriovenous malformation (AVM), aneurysm or vasculitis (fig $1 \mathrm{~B}$ ), as did a subsequent angiogram six weeks later. Magnetic resonance imaging of the brain demonstrated the haematoma but once again no evidence of vasculitis or AVM (fig 1A). He has since received intense neurophysiotherapy, speech therapy, and occupational therapy. He continues to make a gradual recovery with residual right sided weakness, and a mild receptive and expressive dysphasia.

\section{Cerebral haemorrhage}

Cerebral haemorrhage as a complication of CSS has been described but is rare. This case is unique in that there was no documented evidence of cerebral vasculitis, AVM, cerebral aneurysm or hypertension. Liou described a similar case in a patient with uncontrolled blood pressure. ${ }^{39}$ Chang in a case report described intra-ventricular and subarachnoid haemorrhage resulting from necrotising vasculitis of the choroid plexus. ${ }^{47}$ Lanham, $^{3}$ in a series of 16 patients, reported $25 \%$ of cases of CNS disease but did not specify cerebral haemorrhage as a cause.

The cause of cerebral haemorrhage in this case may have been attributable to isolated areas of vasculitis not demonstrated on MRI or cerebral angiography but there is no conclusive proof as to its aetiology.

\section{Further treatment and progress}

After his cerebral haemorrhage a decision was made to treat with further immunosuppression and a course of intermittent intravenous cyclophosphamide, at a dose of $10 \mathrm{mg} / \mathrm{kg}$, was started. To date the patient has not suffered a relapse of vasculitis and has tolerated immunosuppression with no iatrogenic problems.

\section{Discussion}

GENERAL FEATURES OF CSS

CSS is of unknown aetiology. It was first described by Churg and Strauss in 1951 from a series of necropsy findings ${ }^{29}$ and the pathogenesis is believed to be a proliferation of activated eosinophils producing a cascade of aberrant tissue responses. ${ }^{19}$ CSS is increasingly being recognised but epidemiological studies are sparse. Case series have been published that provide valuable information on the features of the disease. ${ }^{1329}$ The incidence was found to be $1.3 \%$ in a large collection of patients with vasculitis. ${ }^{41}$ In an epidemiological study of an ethnically homogenous population of 414000 adults from 1988 to 1994 , the annual incidence of CSS was $2.4 /$ million. $^{42}$ There is an equal sex distribution and onset is reported to be between 20 and 40 years of age in the series described by Lanham, but varied with a range of 16-74 in the ACR series (mean age 50 
Table 11990 criteria for the classification of CSS (traditional format), their sensitivity and specificity versus other defined vasculitis syndromes, and features in this reported case (adapted from reference 1)

\begin{tabular}{llll}
\hline Criterion & Sensitivity (\%) & Specificity (\%) & Reported case features \\
\hline Asthma & 100 & 96.3 & Yes \\
Eosinophilia $>10 \%$ & 95 & 96.6 & Yes \\
Neuropathy, mono or poly & 75 & 79.8 & Yes \\
Pulmonary infiltrates, non-fixed & 40 & 92.4 & No \\
Paranasal sinus abnormality & 85.7 & 79.3 & No \\
Extra-vascular eosinophils & 81.3 & 84.4 & No \\
\hline
\end{tabular}

years). The mean age of onset of vasculitis was 38 years in both series. Lanham described a phasic pattern to the presentation of CSS. The pro-dromal phase consists of allergic disease consisting of asthma, often preceded by allergic rhinitis. The second phase is characterised by peripheral blood eosinophilia and eosinophilic tissue infiltrates. The infiltrative disease may recur over a period of years, but is often interrupted by a life threatening vasculitic phase of the illness. The patient described in this report seemed to follow this pattern with a history of asthma and allergic rhinitis since childhood before the more systemic manifestations of the disease.

DIAGNOSTIC CRITERIA

The ACR and Lanham criteria for the diagnosis of CSS are widely accepted. ${ }^{13}$ Using the ACR criteria, the diagnosis can be made by the traditional method of fulfilling a proportion of criteria from a list or by using the classification tree method of fulfilling a progression of criteria. The latter method starts with the presence of asthma as the most important criterion and is proceeded by eosinophilia of $>10 \%$ and history of allergy. By this method sensitivity was $95 \%$ and specificity $99.2 \%$ when compared with other forms of vasculitis.

Table 1 shows the features used in the traditional method. Four positive of the six listed criteria constitutes a diagnosis of CSS with a sensitivity of $85 \%$ and a specificity of $99.7 \%$.

\section{TREATMENT}

Corticosteroids are the mainstay of treatment in CSS. Corticosteroids alone result in remission of the disease in particular the vasculitis, eosinophilia, IgE, and symptoms of allergic rhinitis and asthma. The response is good but it has been suggested that consideration of immunosuppression, in the form of cyclophosphamide, should depend on prognostic factors. ${ }^{43}$ In addition cyclophosphamide should be reserved for cases in which there is a failure to respond to corticosteroids or disease relapse. Consideration of the organs involved and the severity of their involvement should be taken into account. Factors indicating poor prognosis are renal symptoms (glomerulonephritis or renal insufficiency), gastrointestinal tract involvement, cardiomyopathy, central nervous system involvement, weight loss $>10 \%$ of body weight, or age $>50$ years old. These factors were identified in a retrospective cohort of 165 patients studied. ${ }^{43}$ In a review of 45 cases by Fortin, renal and cardiac involvement were confirmed as poor prognostic factors in systemic necrotising vasculitis. ${ }^{44}$ The possible
Key messages

- Incidence-1. $3 \%$ of patients with vasculitis

- Prevalence-2.4/million in general population

- Main clinical features

Asthma/allergic rhinitis

Neuropathy

Arthralgia

Skin involvement

- Laboratory features Eosinophilia

Raised IgE

- Treatment Corticosteroids

Cyclophosphamide may be required

- Prognosis

Five year survival approximately 62 to $75 \%$

role of plasma exchange was explored by Guillevin et al in a prospective randomised trial with 62 patients. ${ }^{45}$ They found that combined treatment with prednisone, cyclophosphamide, and plasma exchanges, was not superior to prednisone and cyclophosphamide alone in patients with factors indicating poor prognosis.

OUTCOME

The reported survival rate in CSS has been reported as $90 \%$ at one year, and $62 \%$ at five years, by Chumbley. ${ }^{2}$ Guillevin $^{46}$ reported a $75 \%$ five year survival. Compared with PAN, the outcome in CSS is favourable. A retrospective data review of 13 patients with PAN and 12 patients with CSS showed that patients with CSS had a lower mean damage index score, a lower disability score and a higher pain score. ${ }^{47}$ CSS patients had fewer relapses and a lower mortality rate.

\section{SUMMARY}

We have provided a review of the many clinical and laboratory features with which CSS could present. In addition, we have cited the devastating but rare complication of cerebral haemorrhage and the need for immunosuppression to control disease activity to prevent such life threatening consequences.

1 Masi AT, Hunder GG, Lie JT, et al. The American college of rheumatology 1990 criteria for the classification of ChurgStrauss syndrome (Allergic granulomatosis and angiitis). Arthritis Rheum 1990;33:1094-100.

2 Chumbley LC, Harrison EG, De Remee RA. Allergic granulomatosis and angiitis (Churg-Strauss syndrome). Mayo Clin Proc 1977;52:477-84.

3 Lanham JG, Elkon KB, Pusey CD, Hughes GR. Systemic vasculitis with asthma and eosinophilia: A clinical approach vasculitis with asthma and eosinophilia: A clinical approach

to the Churg-Strauss syndrome. Medicine 1984;63:65-81.
4 Lhote F, Guillevin L. Polyarteritis nodosa, microscopic polyangiitis, and Churg-Strauss syndrome. Clinical aspect and treatment. Rheum Dis Clin North Am 1995;21:91146.

5 Yokoyama A, Kohno N, Fujino S, et al. IgG and IgM rheumatoid factor levels parallel interleukin-6 during the vasculitic phase in a patient with Churg-Strauss syndrome. Intern Med 1995;34:646-8.

6 Davies L, Spies JM, Pollard JD, McLeod JG. Vasculitis confined to peripheral nerves. Brain 1996;119:1441-8.

7 Shintani S, Tsuruoka S, Yamada M. Churg-Strauss syndrome associated with third nerve palsy and mononeuritis multiplex of the legs. Clin Neurol Neurosurg 1995; euritis multin

8 Anderson G, Coles ET, Crane $M$, et al. Wegener's granuloma. A series of 265 British cases seen between 1975
graner's and 1985. A report by a subcommittee of the British Thoracic Society Research Committee. Q J Med 1992;83. 427-38. 
9 Martinez-Osuna $\mathrm{P}$, Wallach $\mathrm{P}$, Seleznick $\mathrm{MJ}$, et al. Treatment of the eosinophilia-myalgia syndrome. Semin Arthritis Rheum 1991;21:110-21.

10 Falanga V. Localised scleroderma. Med Clin North Am 1989;73:1143-56.

11 Dawes PT, Smith DH, Scott DL. Massive eosinophilia in rheumatoid arthritis: report of 4 cases. Clin Rheumato 1986;5:62-5

12 Panush RS, Franco AE, Schur PH. Rheumatoid arthritis associated with eosinophilia. Ann Intern Med 1971;75: 199-205.

13 Crisp AJ, Armstrong RD, Grahame R, Dussek JE. Rheumatoid lung disease, pneumothorax, and eosinophilia. Ann toid lung disease, pneumoth

14 Espino-Montoro A, Medina M, Marin-Martin J, et al. Idiopathic eosinophilic myositis associated with vasculitis Idiopathic eosinophilic myositis associated with vasculitis and sym.

15 Bain GA, Flower CD. Pulmonary eosinophilia. Eur J Radiol 1996;233-8.

16 Meeker DP. Pulmonary infiltrates and eosinophilia revisited Cleve Clin J Med 1989;56:199-211.

17 Enright T, Chua S, Lim DT. Pulmonary eosinophilic syndromes. Ann Allergy 1989;62:277-83.

18 Manger BJ, Krapf FE, Gramatzi M, et al. IgE-containing circulating immune complexes in Churg-Strauss vasculitis. Scand J Immunol 1985;21:369-73.

19 Schwartz RA, Churg J. Churg-Strauss syndrome. Br J Dermatol 1992;127:199-204.

20 Finan FC. Rheumatoid papule, cutaneous necrotising granuloma, and Churg Strauss granuloma: are they the granuloma, and Churg Strauss granuloma: are they

21 Wright LF, Bicknell SL. Systemic necrotising vasculitis presenting as epididymitis. J Urol 1986;136:1094.

22 Shurbaji MS, Epstein JI. Testicular vasculitis: implication for systemic disease. Human Pathol 1988;19:186-9.

23 Kroegel C, Costabel U, Guzman J Hirsch F, Ruffle KH, Matthys H. Systemic narcotising vasculitis with bronchia asthma and eosinophilia: Churg-Strauss syndrome. Dtsch Med Wochenschr 1988;113:212-17.

24 Morgan JM, Raposo L, Gibson DG. Cardiac involvement in Churg-Strauss syndrome shown by echocardiography. B Heart J 1989;62:462-6.

25 Leung WH, Wong KK, Lau CP, et al. Myocardial involvement in Churg-Strauss syndrome: the role of involvement in Churg-Strauss syndrome: the role

26 Renaldini E, Spandrio S, Cerudelli B, Affatato A, Balestrier GP. Cardiac involvement in Churg-Strauss syndrome:
follow-up of three cases. Eur Heart J 1993;14:1712-16.

27 Kozak M, Gill EA, Green LS. The Churg-Strauss syndrome. A case report with angiographically documented coronary involvement and a review of the literature. Chest 1995;107:578-80.

28 Renaldini E, Spandrio S, Cerudelli B, et al. Cardiac involvement in Churg Strauss syndrome: A follow-up of three cases. Eur Heart J 1993;14:1712-16.

29 Churg J, Strauss L. Allergic granulomatosis, allergic angiitis and periarteritis nodosa. Am J Pathol 1951;27:277-301.

30 Hasley PB, Follansbee WP, Coulehan JL. Cardiac manifestations of Churg-Strauss syndrome: report of a case and tations of Churg-Strauss syndrome: report of a case

31 Guillevin L, Lhote F, Gallais V, Jarrousse B, et al. Gastrointestinal tract involvement in polyarteritis nodosa and testinal tract involvement in polyarteritis nodosa and 260-7.
32 Gaskin G, Clutterbuck EJ, Pusey CD. Renal disease in the Churg-Strauss syndrome. Contrib Nephrol 1991;94:58.

33 Guillevin L, Lhote F, Amouroux J, et al. Anti-neutrophil cytoplasmic antibodies, abnormal angiograms and pathological findings in polyarteritis nodosa and Churg-Strauss syndrome: indications for the classification of vasculitides of the polyarteritis nodosa group. Br J Rheumatol 1996;35: of the polya

34 Kallenberg CGM, Brouwer E, Weening JJ, et al. Antineutrophil cytoplasmic antibodies: Current diagnostic and pathophysiological potential. Kidney Int 1994;46:1-15.

35 Guillevin L, Visser H, Noel LH, et al. Anti-neutrophil cytoplasm antibodies in systemic polyarteritis nodosa with and without hepatitis $\mathrm{B}$ virus infection and Churg-Strauss syndrome: 62 patients. J Rheumatol 1993;20:1345.

36 Cohen Tervaert JW, Kallenberg C. Neurologic manifestations of systemic vasculitides. Rheum Dis Clin North Am 1993;19:913.

37 O’Donoghue DJ, Nusbaum P, Halbwachs-Mercarelli L, et al. Anti-neutrophil cytoplasmic antibodies associated with polyarteritis nodosa, Churg-Strauss syndrome and HIV related systemic vasculitis. [Abstract]. Am J Kidney Dis 1991;18:208.

38 Gaskin G, Savage COS, Ryan JJ, et al. Anti-myeloperoxidase antibodies in vasculitis: relationship to ANCA and clinical diagnosis. APMIS 1990;98 (suppl 19):33.

39 Liou HH, Liu HM, Chiang IP, Yeh TS, Chen RC. Churg-Strauss syndrome presented as multiple intracerebral haemorrhage. Lupus 1997;6:279-82.

40 Chang Y, Kargas SA, Goates JJ, Horoupian DS. Intraventricular and subarachnoid hemorrhage resulting from necrotizing vasculitis of the choroid plexus in a patient with necrotizing vasculitis of the choroid plexus in a patient with
Churg-Strauss syndrome. Clin Neuropathol 1993;12: Churg-

41 Lie JT. The classification of vasculitis and a reappraisal of granulomatosis angiitis (Churg-Strauss syndrome). Mt Sinai J Med 1986;53:429-39.

42 Watts RA, Carruthers DM, Scott DG. Epidemiology of systemic vasculitis: changing incidence or definition? Semin Arthritis Rheumat 1995;25:28-34.

43 Guillevin L, Le Thi Huong D, Godeau P, et al. Clinical findings and prognosis of polyarteritis nodosa and ChurgStrauss angiitis: a study of 165 patients. $\mathrm{Br}$ J Rheumatol 1988;27:258-66.

44 Fortin PR, Larson MG, Watters AK, et al. Prognosis factors in systemic necrotising vasculitis of the polyarteritis nodosa group. A review of 45 cases. J Rheumatol 1995;22: 78-84.

45 Guillevin L, Lhote F, Cohen P, et al. Corticosteroids plus pulse cyclophosphamide and plasma exchanges versus corticosteroids plus pulse cyclophosphamide alone in the treatment of polyarteritis nodosa and Churg-Strauss treatment of polyarteritis nodosa and Churg-Strauss syndrome patients with factors predict

46 Guillevin L, Guittard T, Bletry O, et al. Systenfic narcotising angiitis with asthma: causes and precipitating factors in 43 cases. Lungs 1987;165:165-72.

47 Abu-Shakra M, Smythe H, Lewtas J, et al. Outcome of polyarteritis nodosa and Churg-Strauss syndrome. An analysis of 25 patients. Arthritis Rheum 1994;37:1798-803. 